\title{
Nachhaltig managen mit der Balanced Scorecard
}

\author{
Wie kann unternehmerische Nachhaltigkeit umgesetzt und in das allgemeine \\ Managementsystem von Unternehmen integriert werden? Ein mögliches Instru- \\ ment und Methodik ist die so genannte Balanced Scorecard (BSC). In einem \\ jïngst abgeschlossenen Projekt wurde die Entwicklung von Sustainability BSCs \\ und die Integration von Nachhaltigkeitszielen in bereits bestehende BSCs in der \\ Praxis erprobt (1).
}

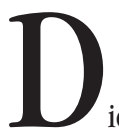

\section{Von C. U. Gminder, T. Bieker,}

T. Hahn und M. Wagner lung bereitet vielen Unternehmen Probleme. Von positiven Ausnahmen abgesehen, ist Nachhaltigkeit wenig in die Managementsysteme, -prozesse und -kultur integriert. Können diese Schwierigkeiten durch den Einbezug nachhaltigkeitsorientierter Zielen in die Balanced Scorecard bzw. der Entwicklung einer Sustainability Balanced Scorecard (SBSC) gelöst werden? „Das kommt ganz darauf an", ist unsere Antwort. Sie ist typisch für solcherlei anwendungsorientierte empirische Forschung. Denn in den Partnerunternehmen Axel Springer Verlag, Berliner Wasserbetriebe, Flughafen Hamburg, OBI Baumärkte, Volkswagen und Unaxis wurden verschiedene Erfahrungen gesammelt. Diese wurden in vertieften Einzelfallstudien qualitativ analysiert und interpretativ ausgewertet. Hieraus lassen sich Faktoren ableiten, die bei der Entwicklung einer SBSC besonders zu beachten sind, weil sie eine fördernde oder hemmende Wirkung entfalten.

\section{- Strategische Faktoren}

Die Balanced Scorecard ist ein Kennzahlen- und Managementsystem zur Umsetzung von Strategien. Eine SBSC setzt also nachhaltigkeitsorientierte Strategien voraus. Diese sind selten explizit vorhanden, wie die empirische Forschung zeigt und wie sich im Forschungsprojekt bestätigte (2). Deshalb ist es zunächst nötig, Nachhaltigkeitsstrategien zu definieren und daraus strategische Ziele abzuleiten. Die Klärung der Strategie wird dadurch zu einem Element der SBSC-Entwicklung. Dabei ist die Forderung nach einer expliziten Nachhaltigkeitsstrategie ein anspruchsvolles Ziel. Doch sie löst Diskussionen aus, durch die das
Verhältnis der drei Nachhaltigkeitsdimensionen sowie die langfristige Relevanz von Umwelt- und Sozialaspekten für Unternehmens- und Geschäftsfeldstrategie geklärt werden können.

In Bezug auf die Umsetzung von Strategien wurden widersprüchliche Erfahrungen in den Partnerfirmen gemacht. Es gelingt nicht immer die Lücke zwischen Strategie und Umsetzung zu schließen. Auf jeden Fall aber scheint die Lücke kleiner zu werden. Dies liegt vor allem daran, dass Leitsätze, Strategien und Visionen zu unspezifisch formuliert sind, um für die Zielformulierung der SBSC prägend sein zu können. Eine Ableitung von Zielen aus den Strategien hat in den Fallfirmen auch nicht immer stattgefunden. In manchen Fällen erfolgte eine nachträgliche Verknüpfung von SBSC-Zielen mit der Strategie, um den Ansprüchen der Leitung zu genügen.

\section{- Kulturelle Faktoren}

Kulturelle Faktoren spiegeln die vorherrschenden Prägungen, Denk- und Verhaltensweisen in einem Unternehmen wider. Von ihnen hängen maßgeblich Akzeptanz und Verständnis und somit der Erfolg eines neuen Managementinstruments ab. Bei der Sustainability BSC kommen zur neuen Methodik auch neue Inhalte hinzu. Bezüglich der Methodik ist wichtig, welche Einstellungen zum strategischen, zielorientierten Denken und zum transparenz- und kennzahlenorientierten Controlling existieren. Bezüglich des Inhalts ist es wichtig, inwiefern die Unternehmenskultur einer nachhaltigen Entwicklung Raum gibt. Je bedeutsamer Nachhaltigkeit im Selbstverständnis des Unternehmens verankert ist, desto größer ist die Aufgeschlossenheit gegenüber neuen Ansätzen des Nachhaltigkeitsmanagements. Dies gilt insbesondere außerhalb der Umweltschutzabteilung, da mit der SBSC eine Integration von Umwelt- und Sozialaspekten in die ökonomischen Kernaktivitäten angestrebt wird. Wird beispielsweise Umweltschutz prozessorientiert als Verantwortung aller Mitarbeiter betrachtet, erleichtert dies eine SBSC. Ist er fachspezifisch konzentriert und stärker an der technischen Machbarkeit als an der strategischen Relevanz ausgerichtet, erschwert dies eine SBSC. Je stärker das existierende Managementverständnis mit den inhaltlichen und methodischen Merkmalen der SBSC übereinstimmt, desto niedriger die Hürden für eine Einführung.

Lernbereitschaft und Offenheit sind bedeutende kulturelle Grundlagen, vor allem auch die Fähigkeit zum zielorientierten Arbeiten und zum Umgang mit der Transparenz, welche das Messen von Zielen schafft. Die Formulierung einer SBSC erfordert auch die Bereitschaft, bestehende Aktivitäten und Schwerpunktsetzungen kritisch zu hinterfragen und zu überdenken. Eine offene, konstruktive Diskussionskultur über Abteilungsgrenzen und Fachgebiete hinweg ist zudem einer SBSC förderlich.

\section{Mikropolitische Faktoren}

Die Entwicklung und Einführung jeder BSC geht unweigerlich mit mikropolitischen Prozessen im Unternehmen einher. Hier stehen Faktoren wie Handlungsspielraum, Macht, Prestige und Karriereentwicklung von individuellen Akteuren oder ganzen Abteilungen im Vordergrund. Für eine SBSC hat die persönliche Wertschätzung ökologischer und sozialer Nachhaltigkeit durch die Prozessbeteiligten besonderes mikropolitisches Gewicht, allen voran durch die „Mächtigen“. Interessant sind hierbei die Diskrepanzen zwischen Äußerungen im Einzelgespräch und in den Workshop-Diskussionen. Häufiger wurde mit persönlichem Bedauern darauf hingewiesen, dass ,im Unternehmen“ nur kurzfristig finanziell lohnenswerte Beiträge akzeptiert würden. Deshalb wurde versucht, den kleinsten gemeinsamen Nenner zu antizipieren. Es zeigt sich, dass vor einer Integration ökologischer und sozialer Aspekte in die BSC zunächst eine Integration in den Köpfen der Mitarbeiter und Manager stattfinden muss. Besondere mikropolitische Rollen nehmen daher die Führungskräfte für die Akzeptanz sowie die Umwelt- und Sozialmanager für die Entwicklung der SBSC ein. Letztere müssen vor allem bereit sein, die tatsächliche Stellung ihres Themas im Unternehmen wahrzunehmen und zu akzeptieren, dass Umwelt- und Sozialziele im Rahmen eines integrierten Vorgehens nicht mehr 
von ihnen alleine bestimmt und gesteuert werden. Der damit einhergehende Macht- und Unabhängigkeitsverlust kann - wie in einem Partnerunternehmen geschehen - sogar zur Ablehnung einer SBSC führen.

\section{- Prozessbezogene Faktoren}

Prozessbezogene Faktoren beziehen sich zum einen auf ein gekonntes Projektmanagement, zum anderen auf die Art und Weise, in der die Verbindung zur Strategie hergestellt und die SBSC eingefuihrt wird. Die BSC setzt eine top-down Logik voraus. In der Praxis stößt dieser planerische Ansatz häufig auf Skepsis und Widerstand, weil er die bisherigen Tätigkeiten und entsprechend verteilte Budgets in Frage stellt. Erfasst er die Komplexität aller Anforderungen und Ziele? Ist er nicht geschichts- und damit erfahrungsblind? Unserer Erfahrung nach bietet es sich daher an, in einem Gegenstromverfahren ,top-down“ und ,,bottomup“ zeitversetzt miteinander zu verknüpfen. Der strategische Input wird als Rahmen top-down vorgegeben; die Ziele, Maßnahmen und Kennzahlen werden durch untere Managementebenen bottom-up ausgefüllt und zurückgespielt. Gerade beim Thema Nachhaltigkeit ist der Einbezug aller Ebenen wichtig, um die breite Umsetzung zu gewährleisten. Allerdings dauert der Prozess damit länger und ist diskussionsintensiver.

\section{- Strukturelle Faktoren}

Diese ergeben sich aus dem Einbezug in das allgemeine Managementsystem. Gibt es bereits eine „klassische“ BSC, in die ökologische und soziale Aspekte integriert werden sollen? Oder wird eine SBSC entworfen, die alle drei Nachhaltigkeitsdimensionen von Anbeginn berücksichtigt? Bei der Neuentwicklung sollten andere Systeme durch die SBSC abgelöst werden. Andernfalls resultiert ein Zusatzaufwand, der die Akzeptanz senkt. Existiert dagegen bislang kein Managementsystem, füllt die SBSC eine Lücke. Künftige SBSC-Projekte sollten daher großen Wert auf eine explizite und möglichst substituierende Verankerung im ,Instrumentenkasten“ des Managements legen. In folgenden Managementprozessen sollte die SBSC verwendet werden, um ihr Lebensfähigkeit zu verschaffen: Zum Ersten in den Geschäftsprozessen als Steuerungsinstrument; zum Zweiten in den Review-Prozessen, in denen sie - zumeist jährlich - überarbeitet wird. Zum Dritten sollten andere Prozesse wie Strategie- und Zielfindung, Budgetierung, Gehaltsfestlegung etc. mit der
SBSC verknüpft sein. Denn welches Instrument wird ,gelebt“, wenn es in keinem Managementprozess verwendet wird, sondern isoliert ist? Bei der SBSC muss zusätzlich das Umwelt- und Sozialmanagement berïcksichtigt werden. Dort werden spezielle Instrumente eingesetzt, wie Umweltmanagementsysteme nach ISO 14001 oder EMAS. Und es laufen spezielle Managementprozesse ab, wie Planung, Ausfiuhrung, Kontrolle (Audits) und Berichterstattung. Die SBSC sollte auch hier nicht additiv eingesetzt werden. Schließlich ist Ziel einer SBSC die Integration von ökologischer und sozialer Nachhaltigkeit in das allgemeine Managementsystem. Mit der Entwicklung einer SBSC müssen daher die Umwelt- und Sozialmanager zwei meist parallel laufende Managementwelten zusammenführen, ihre eigene und die klassisch finanzielle.

\section{- Methodische Faktoren}

Methodisch ist die Gestaltung der einzelnen BSCElemente wichtig. Bei der Zahl der Ziele lässt sich durchweg die Praxisregel „twenty is plenty“ empfehlen. Die mit der SBSC-Entwicklung einhergehende Zielreduktion wird zunächst als schmerzlich, aber dann als sehr befreiend empfunden. Die Konzentration auf das Wesentliche wird möglich. Gleiches gilt für die Anzahl der Kennzablen zur Zielunterstuitzung (Einflusskennzahlen) und Messung der Zielerreichung (Ergebniskennzahlen). Allerdings ist die Formulierung geeigneter Kennzahlen eine Kunst und - aus mikropolitischen Gründen - nicht immer erwünscht. Zusätzlich können methodische Probleme auftreten, wie die Frage der Operationalisierbarkeit von Zielen, die Aussagekraft, die Mess- und Beeinflussbarkeit von Kennzahlen.

Eine große methodische Stärke der BSC liegt im Herausarbeiten von Ursache-Wirkungsbeziebungen zwischen den verschiedenen Zielen. Sie leisten einen wertvollen Beitrag zur Zusammenfassung und Verknüpfung der Überlegungen und werden in Form einer Strategy Map veranschaulicht. Durch Zahl und Richtung der Verknüpfungen kann die relative Bedeutung der jeweiligen Ziele erkannt werden. Im Kontext der Nachhaltigkeit ist es auch hilfreich, Zielkonflikte offen zu legen und zu diskutieren. Somit stellt die SBSC eine geeignete Methodik dar, um Dilemma-Situationen zwischen den einzelnen Dimensionen der Nachhaltigkeit aufzuzeigen. Bezüglich der Frage, welche und wie viele Perspektiven in einer SBSC definiert werden, bestätigt sich die Regel der individuellen Anpassung einer BSC an die strategischen Gegebenheiten.
Wichtig ist der Klärungsprozess, der mit der Bündelung der Ziele in Perspektiven einhergeht. Sind alle strategischen relevanten Stakeholder mit den Zielen erfasst worden? Oder gibt es noch „,blinde Flecken“? In Bezug auf Nachhaltigkeit kann eine zusätzliche Perspektive hilfreich sein, um im nichtmarktlichen Bereich der gesellschaftlichen Verantwortung Ziele abzubilden.

\section{$>$ Fazit}

Eine Sustainability Balanced Scorecard bietet die Chance für das Umwelt- und Sozialmanagement, einen großen Schritt zur Integration in das allgemeine Managementsystem zu machen. Der Entwicklungsprozess einer SBSC stellt allerdings einen komplexen unternehmenspolitischen Prozess dar. Er erfordert Geduld, Stehvermögen und unternehmensinterne Promotoren und Triebkräfte. Gelingt es den Nachhaltigkeitspromotoren, sich eröffnende Möglichkeiten (,Windows of Opportunity“) im richtigen Moment zu nutzen, kann Nachhaltigkeit im alltäglichen Management verankert werden. Ausdauer ist hier wichtig, da dieser Prozess nicht immer geradlinig und einfach verläuft. Es ist wie beim Segeln: Man kommt bei Gegenwind nur durch häufiges Wenden und Kreuzen in einer Zick-Zack-Linie voran.

\section{Anmerkungen}

(1) Die im Folgenden zusammengefassten Ergebnisse wurden im zweijährigen BMBF- Forschungsprojekt Sustainability Balanced Scorecard (SBSC) der Universitäten Lüneburg und St. Gallen gewonnen. Vgl. ausführlich Schaltegger, Stefan/ Dyllick, Thomas (Hrsg.): Nachhaltig managen mit der Balanced Scorecard, Wiesbaden 2002.

(2) Dyllick, Thomas/ Schaltegger, Stefan: Nachhaltigkeitsmanagement mit einer Sustainability Balanced Scorecard. In: UmweltWirtschaftsForum, Nr. 4/2001, S. 68-73.

\section{Die Autoren}

Thomas Bieker und Carl Ulrich Gminder sind wissenschaftliche Mitarbeiter am Institut für Wirtschaft und Ökologie der Universität St. Gallen. Kontakt: IWÖ-HSG, Tigerbergstr. 2, CH-9000 St. Gallen. Tel. 0041-71-224-2744, E-Mail: thomas.bieker@unisg.ch, carl-ulrich.gminder@unisg.ch Tobias Hahn und Marcus Wagner sind wissenschaftliche Mitarbeiter und forschen am Centrum für Nachhaltigkeitsmanagement der Universität Lüneburg.

Kontakt: Universität Lüneburg, Scharnhorststr. 1, 21335 Lüneburg. Tel. 04131-78-2216, E-Mail: tobias.hahn@uni-lueneburg.de, marcus.wagner@uni-lueneburg.de 
(c) 20I0 Authors; licensee IÖW and oekom verlag. This is an article distributed under the terms of the Creative Commons Attribution Non-Commercial No Derivates License (http://creativecommons.org/licenses/by-nc-nd/3.o/), which permits unrestricted use, distribution, and reproduction in any medium, provided the original work is properly cited. 